\title{
Brief Communication: Impact of Sign Character Aspect Ratio on Legibility
}

\section{John D. Bullough, Ph.D.}

Lighting Research Center, Rensselaer Polytechnic Institute

Troy, NY

Correspondence: Lighting Research Center, Rensselaer Polytechnic Institute, 21 Union Street, Troy, NY 12180 USA.

Email: bulloj@rpi.edu, Tel.: +1.518.276.7100,

Fax: +1.518.276.7199

\section{INTRODUCTION}

The ability for pedestrians, drivers, cyclists and other road and sidewalk users to identify and read signs is crucial for safe and efficient navigation through the built exterior environment. A good deal of effort has been made regarding the legibility of alphanumeric characters that might be used on signs (Tinker, 1963). Quantitative models of visual performance (e.g., Rea \& Ouellette, 1991) to predict the relative speed and accuracy with which people can identify visual information on a sign include factors such as the luminance of the sign, the contrast between the characters on a sign and their background, and the size of the characters.

Empirical studies of the readability of different typefaces have also been conducted (Garvey et al., 1997; 2001; 2004; 2016). For example, investigations of typeface characteristics that improve legibility have indicated that often there are few, if any, differences between serif and non-serif fonts in terms of readability (Carter, Day, \& Meggs, 1985; Kuhn, Garvey, \& Pietrucha, 1998). A factor that has not been evaluated in many studies of legibility is the aspect ratio of a font's characters. Some published guidelines (e.g., CIDEA, 2010) suggest that a character on a sign is maximally legible when its height and width are nearly the same. There is indirect evidence supporting this claim; Bullough (2016) used the relative visual performance (RVP) model (Rea \& Ouellette, 1991) to predict the legibility of highway sign characters with different font characteristics in a study by Garvey et al. (2016). Legibility distances were generally related to the size of the characters when luminance and contrast were held constant, but were systematically shorter when the sign characters were narrowest

\section{Abstract /}

Signs are critical elements of the visual environment for pedestrians, drivers and other users. Regardless of their purpose, signs should be easily detected and readily legible. Tools such as visual performance models have been developed to allow the assessment of the speed and accuracy with which observers can read information on a sign. These models include factors such as the luminance of the sign, the contrast between the sign's characters and their backgrounds, and the size of the characters. One factor that has not been included in visual performance models is the aspect ratio of characters, particularly of alphanumeric symbols. To address this, a small study was carried out to investigate observers' abilities to identify characters ranging in contrast and aspect ratio. 
in terms of aspect ratio. The present paper summarizes a pilot laboratory study conducted to validate the post hoc analysis from Bullough (2016).

\section{METHOD}

Ten individuals aged 21 to 47 years (mean 37) participated in the study. Participants took part in a series of experimental trials, each consisting of viewing a random five-digit number for 2 seconds in the center of a computer display screen with a background luminance of $100 \mathrm{~cd} / \mathrm{m}^{2}$. After the 2-second interval, the screen was blanked and four random five-digit numbers, one of which was the number previously displayed, were shown at the top, bottom, left, and right sides of the display screen. The location of the correct number was randomized for each trial. Participants were asked to indicate, as quickly as possible, the location of the number that they had previously seen, by pressing the appropriate arrow key on a computer keyboard.

The aspect ratio of the characters was defined as the ratio between the height and width of the numerical symbols, and was $5.25,1.26,0.78,0.46$, or 0.26 for each trial (see Figure 1). All of the characters, regardless of their aspect ratio, subtended the same solid angle so that their size would be predicted to be the same by the RVP model (Rea \& Ouellette, 1991).

The luminance contrast $(\mathrm{C})$ of the numbers was defined by the following equation:

$$
\mathrm{C}=\left|\mathrm{L}_{\mathrm{b}}-\mathrm{L}_{\mathrm{c}}\right| / \mathrm{L}_{\mathrm{b}}
$$

where $\mathrm{L}_{\mathrm{b}}$ was the luminance of the background (always $100 \mathrm{~cd} / \mathrm{m}^{2}$ ) and $\mathrm{L}_{\mathrm{c}}$ was the luminance of the characters. The character luminance was either $10 \mathrm{~cd} /$ $\mathrm{m}^{2}$ or $87 \mathrm{~cd} / \mathrm{m}^{2}$, resulting in luminance contrasts of 0.9 (high contrast) or 0.13 (low contrast), as illustrated qualitatively in Figure 2.

Each participant made 100 identification trials. With five aspect ratios and two contrast levels, there were 10 experimental conditions, and participants experienced 10 trials for each condition. All trials and conditions were presented in a randomized order.

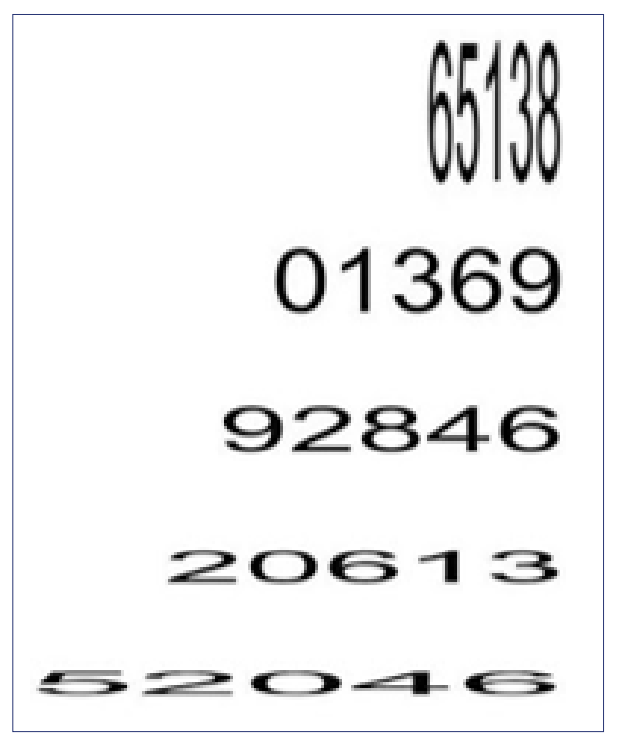

Figure 1 / Character aspect ratios investigated in the legibility study. From top to bottom, aspect ratios (height/width) are 5.25, 1.26, 0.78, 0.46 , and 0.26 .

\section{6}

Figure 2 / Illustration of luminance contrast values used in the legibility study. The contrast $(\mathrm{C})$ at left is 0.9 ; the contrast at right is 0.13 . (Exact contrasts might not match what was displayed during the actual experiment.) 


\section{RESULTS}

Accuracy of identification was always at least 96\%. The identification times (Figure 3) were statistically significantly impacted by both contrast $\left(\mathrm{F}_{1,9}=106, \mathrm{p}<\right.$ $0.001)$ and the aspect ratio $\left(\mathrm{F}_{4,36}=3.99, \mathrm{p}<0.01\right)$, based on a within-subjects analysis of variance (ANOVA), and there was no statistically significant interaction $\left(F_{4,36}=0.38, p>0.05\right)$ between contrast and aspect ratio.

\section{DISCUSSION}

Visual performance models that use the solid angular size of the object to be seen as the characterization of size, such as the RVP model (Rea \& Ouellette, 1991), would predict all of the aspect ratios in the present study to have the same size, but the results in Figure 3 suggest that very narrow or wide characters are not identified as quickly as those with aspect ratios closer to one. Of interest however, the RVP model predicts (for a 37-year-old observer, the mean age of the subjects in this experiment) a visual response time for the low-contrast characters that is $18 \%$ longer than for the high-contrast characters. The average increase in identification times for the low-contrast characters in the present study over the high-contrast characters was also $18 \%$.

This correspondence supports the notion that the RVP model, which allows the user to estimate visual response times based on light level, size, and contrast (Rea \& Ouellette, 1991), can be a useful tool in assessing the legibility properties of sign characters, provided differences in character aspect ratio are also considered.

The RVP model could, therefore, be used to assess the relative impacts of different aspect ratios in terms of differences in contrast. For example, the optimal aspect ratio in the present study was 1.26 , whereas the aspect ratio (among the ones tested) that elicited the longest identification times was 0.26 . On average, characters with an aspect ratio of 0.26 had identification times that were 14\% longer than those with an aspect ratio of 1.26. Using the RVP model (assuming the same character size and observer average age as in the experiment), it can be determined that the luminance contrast reduction that results in a $14 \%$ increase in visual response time is a reduction from 0.9 to 0.16 .

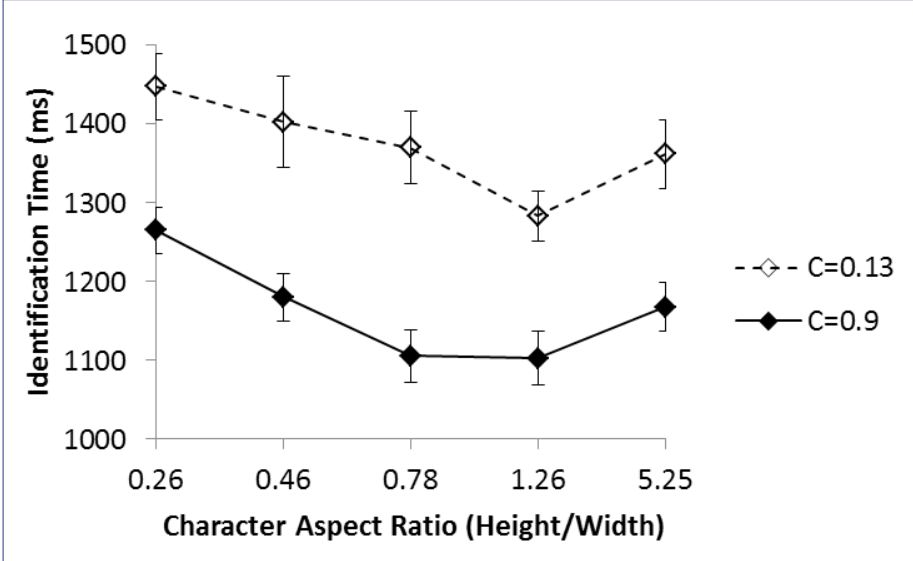

Figure 3 / Mean identification times ( \pm standard error of the mean) for the five-digit numbers as a function of contrast and character aspect ratio.

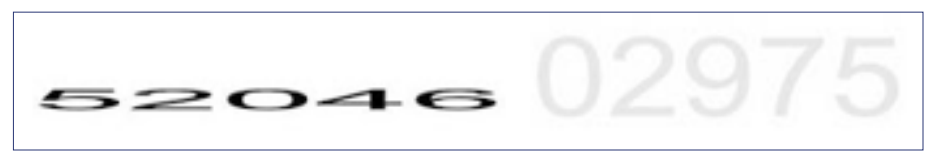

Figure 4 / Left: Characters with a contrast of 0.9 and an aspect ratio of 0.26 . Right: Characters with a contrast of 0.16 and an aspect ratio of 1.26. Both sets of characters would be expected to be equally legible based on the present results. (Exact contrasts may not match the stated values.) 
In other words, under the conditions of the present experiment, characters with a contrast of 0.9 and an aspect ratio of 0.26 are equally legible (if legibility means being able to quickly identify characters) to characters with a contrast of 0.16 and an aspect ratio of 1.26. Figure 4 illustrates these conditions that would be expected to result in equal legibility.

\section{CONCLUSION}

The results of this pilot study, although limited by a small participant sample size and relatively young observers, clearly demonstrate a systematic effect of aspect ratio in the legibility of characters. They also illustrate the utility of models such as the RVP model (Rea \& Ouellette, 1991) to provide quantitative assessments of the legibility of sign characters while simultaneously pointing out an important shortcoming, especially when typefaces with aspect ratios deviating from 1 are involved. These results suggest however, as illustrated in Figure 4, that very narrow or very wide typefaces could be addressed by a correction factor that trades off contrast with aspect ratio.

\section{ACKNOWLEDGMENTS}

Preparation of this manuscript was supported by the Sign Research Foundation under the project "Illuminated Sign Conspicuity: What Factors Make a Sign Noticeable and Legible," managed by Sapna Budev. Helpful input and assistance in this study was provided by Matthew Tice, Nicholas Skinner, Chris Gaudette, David Hickey, Kenneth Peskin, Deacon Wardlow, and John Yarger.

\section{REFERENCES}

Bullough, J.D. (2016). Discussion of "Legibility of the Clearview typeface and FHWA standard alphabets on negative- and positivecontrast signs" by Garvey et al. Transportation Research Record, 2555, 36-37.

Carter, R., Day, B., \& Meggs, P. (1985). Typographic design: Form and communication. New York, NY: Van Nostrand Reinhold.

Center for Inclusive Design and Environmental Access (CIDEA). (2010). Design resources DR-11: Text legibility and readability of large format signs in buildings and sites. Buffalo, NY: University at Buffalo.

Garvey, P.M., Pietrucha, M.T., \& Meeker, D. (1997). Effects of font and capitalization on legibility of guide signs. Transportation Research Record, 1605, 73-79.

Garvey, P.M., Zineddin, A.Z., \& Pietrucha, M.T. (2001). Letter legibility for signs and other large format applications. Proceedings of the Human Factors and Ergonomics Society 45th Annual Meeting, 1443-1447.

Garvey, P.M., Chirwa, K.N., Meeker, D.T., Pietrucha, M.T., Zineddin, A.Z., Ghebrial, R.S., \& Montalbano J. (2004). New font and arrow for National Park Service guide signs. Transportation Research Record, 1862, 1-9.

Garvey, P.M., Klena, M.J., Eie, W.-Y., Meeker, D.T., \& Pietrucha, M.T. (2016). Legibility of the Clearview typeface and FHWA standard alphabets on negative- and positive-contrast signs. Transportation Research Record, 2555, 28-36.

Kuhn, B.T., Garvey, P.M., \& Pietrucha, M.T. (1998). Sign legibility: The impact of color and illumination on typical on-premise sign font legibility. Bristol, PA: United States Sign Council Foundation.

Rea, M.S., \& Ouellette, M.J. (1991). Relative visual performance: A basis for application. Lighting Research and Technology, 23(3), $135-144$.

Tinker, M.A. (1963). Legibility of print. Ames, IA: University of Iowa Press. 